\title{
How to determine the thermal electron density and the magnetic field strength from the Cluster/Whisper observations around the Earth
}

\author{
J. G. Trotignon ${ }^{1}$, P. M. E. Décréau ${ }^{1}$, J. L. Rauch ${ }^{1}$, O. Randriamboarison ${ }^{1}$, V. Krasnoselskikh ${ }^{1}$, P. Canu ${ }^{2}$, H. Alleyne ${ }^{3}$, \\ K. Yearby ${ }^{3}$, E. Le Guirriec ${ }^{1}$, H. C. Séran ${ }^{1}$, F. X. Sené ${ }^{1}$, Ph. Martin ${ }^{1}$, M. Lévêque ${ }^{1}$, and P. Fergeau ${ }^{1}$ \\ ${ }^{1}$ Laboratoire de Physique et Chimie de l'Environnement, Centre National de la Recherche Scientifique, 3 A avenue de la \\ Recherche Scientifique, F-45071 Orléans cedex 02, France \\ ${ }^{2}$ Centre d'Etudes des Environnements Terrestres et Planétaires, Centre National de la Recherche Scientifique, Université \\ Versailles Saint-Quentin-en-Yvelines, 10 avenue de l'Europe, F-78140, Vélizy Villacoublay, France \\ ${ }^{3}$ Space Instrumentation Group, Department of ACSE, University of Sheffield, Mappin Street, Sheffield, S1 3JD, UK
}

Received: 13 April 2001 - Revised: 22 June 2001 - Accepted: 25 June 2001

\begin{abstract}
The Wave Experiment Consortium, WEC, is a highly integrated package of five instruments used to study the plasma environment around the Earth. One of these instruments, the Waves of HIgh frequency and Sounder for Probing of Electron density by Relaxation, Whisper, aims at the thermal electron density evaluation and natural wave monitoring in the $4-83 \mathrm{kHz}$ frequency range. In its active working mode, which is our primarily concern here, the Whisper instrument transmits a short wave train at a swept frequency and receives echoes after a delay. Incidentally, it behaves like a classical ground-based ionosonde. Natural modes of oscillations may thus be excited in the surrounding medium. This means that with suitable interpretations, the Whisper sounding technique becomes a powerful tool for plasma diagnosis. By taking into account the characteristic frequencies of the magnetoplasmas encountered by the Cluster spacecraft, it is indeed possible to reliably and accurately determine the electron density and, to a lesser degree, the magnetic field strength from the Whisper electric field measurements. Due to the predominantly electrostatic nature of the waves that are excited, observations of resonances may also lead to information on the electron velocity distribution functions. The existence of a hot population may indeed be revealed and the hot to cold density ratio can be estimated.
\end{abstract}

Key words. Magnetospheric physics (plasma waves and instabilities). Space plasma physics (active perturbation experiments; instruments and techniques)

\section{Introduction}

For more than 20 years, relaxation sounders have been successfully used for probing plasmas around the Earth (GEOS

Correspondence to: J. G. Trotignon

(jgtrotig@cnrs-orleans.fr)
$1 \& 2$, ISEE-1, Viking), near Jupiter, and in the interplanetary medium (Ulysses). They have indeed proved to be efficient in providing absolute measurements of the total plasma density, natural wave monitoring, and possibly magnetic field intensity determination.

In their active modes, relaxation sounders behave like classical radars. A radio wave transmitter sends a wave train during a very short time period $(\sim 1 \mathrm{~ms})$ at a given frequency. Next, a receiver, usually tuned to the same frequency, listens to the signal induced in the surrounding plasma by means of E-field antennae. The working frequency is then shifted for a new sounding. The process is thus repeated until the full frequency bandwidth is covered.

The sketch shown at the top of Fig. 1 illustrates how the Whisper relaxation sounder operates. The two long double sphere electric antennae of the Electric Field and Wave experiment, EFW (Gustafsson et al., 1997), are needed to transmit and receive the electric field signals used by Whisper. These antennae are shown in the bottom panel of Fig. 1. They consist of four orthogonal cable booms carrying spherical sensors and deployed in the spacecraft spin plane. These electric antennae, designated $E_{Y}$ and $E_{Z}$, have sphere-tosphere separations of $88 \mathrm{~m}$. The $8 \mathrm{~cm}$ in diameter spheres are made of aluminium and are coated by a conductive paint. The high-impedance preamplifiers that are in the hockey pucks, close to the spheres, provide signals to the electronic boards and in particluar, to the Whisper receiver electronic board. The boom cables are composed of several wires, a coaxial, a kevlar braid, and a conductive outer braid. The Whisper transmitter is connected to the outer braids of the $E_{y}$ pair of booms. These braids are displayed in orange in the top sketch of Fig. 1.

The Whisper instrument primarily consists of a pulse transmitter, a sensitive radio receiver, a digital spectrum analyser and a controller unit. For further information and details on the Whisper electronics, the reader is referred to the 


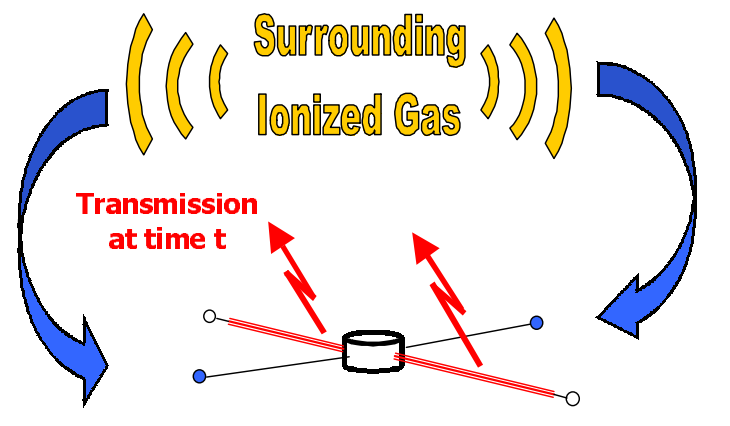

Reception $\begin{array}{ll}\text { at time } t+\Delta t & \begin{array}{l}\text { Plasma frequency } \mathrm{Fp} \\ \text { identified on ground }\end{array} \mathrm{Ne} \alpha \mathrm{Fp}^{2}\end{array}$

\section{CLUSTER S/C}

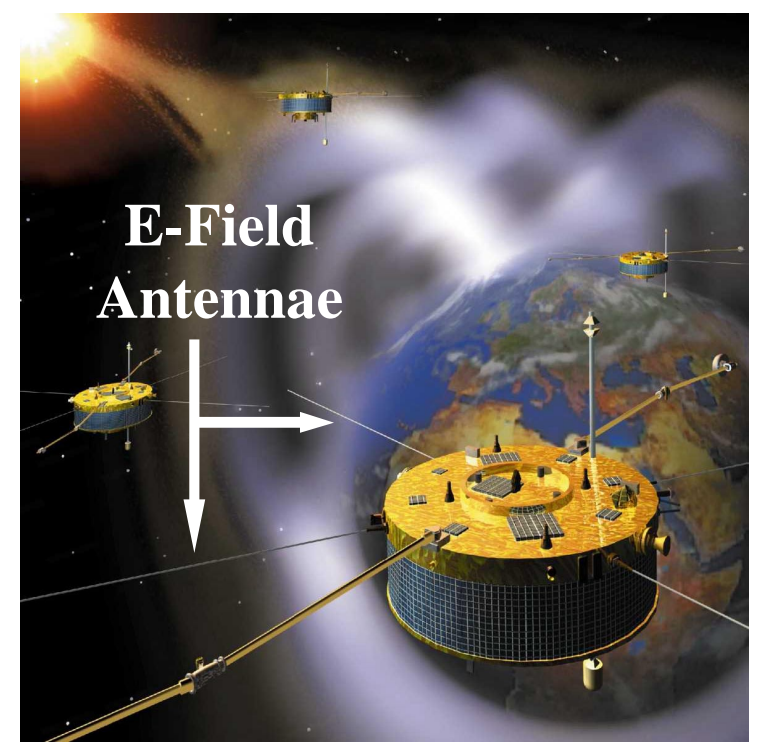

Fig. 1. Whisper relaxation sounder principle of measurements (top), EFW long double sphere electric antennae used by Whisper (bottom).

papers by Décréau et al. (2001, this issue) and Décréau et al. (1997). Let us also recall that Whisper could not properly be monitored without the help of the Digital WaveProcessing experiment, DWP (Woolliscroft et al., 1997), which is part of the Wave Experiment Consortium, WEC (Lefeuvre et al., 1993). Compared to previous relaxation sounders, the Whisper wave analysis is provided by fast Fourier transforms (FFTs) instead of swept frequency analyses (Décréau et al., 1997). In this way, Whisper has new capabilities. Though only six frequency bins are stimulated during one transmitting step, a whole frequency spectrum is computed on board ( 512 bins, $162 \mathrm{~Hz}$ in width). This means that two successive spectra may be used to provide six passive bins and six active bins with a very short time delay, provided that the two successive transmitting pulses are sent at two sufficiently separated frequencies. At the end, when the whole frequency bandwidth is covered, two complete spec- tra, one active and one passive, are available.

Whenever the transmitter is switched off (passive mode), Whisper becomes a simple natural wave receiver. The highfrequency part of the natural electric emissions, from 2 to $80 \mathrm{kHz}$, is then monitored.

Here, we only pay attention to the active response of the Whisper relaxation sounder, from which the electron density and magnetic field strength are derived. The second section of this paper examines the resonances observed by Whisper from the solar wind down to the Earth's magnetosphere close to the plasmapause. Sections 2 and 3 describe how the plasma resonances may be recognized and identified. The presentation then moves on, in Sect. 5, to show some results obtained in the magnetosphere for different electron plasma frequency to electron cyclotron frequency ratios. A plasmapause crossing event is analysed in detail at the end of this section in order to bring the sounder diagnosis power to the forefront. The conclusions are then given in the last section.

\section{Plasma resonances observed by Whisper}

When the transmitted pulse frequency is close to some plasma characteristic frequencies, very intense echoes are received. These stimulated signals are called resonances, and they are strongly monochromatic and correspond to frequencies for which the group velocity is very small and close to the spacecraft velocity (Etcheto et al., 1981; Belmont et al., 1984). Different types of resonances are actually observed in the Earth's environment, depending on the encountered region.

In the magnetosphere, resonances possibly occur at the electron cyclotron frequency, $F_{c e}$, and its harmonics, $n F_{c e}$, as well as at the total plasma frequency, $F_{p e}$, the upper-hybrid frequency, $F_{u h}=\sqrt{ }\left(F_{p e}^{2}+F_{c e}^{2}\right)$, and the Bernstein's mode frequencies, $F_{q n}$ (Bernstein, 1958). The latter are actually the maxima in the Bernstein's mode dispersion curves (see, for example, Fig. 1 in Pottelette et al., 1981). The upper panel in Fig. 2 displays the electric field intensities measured by Whisper on board the Cluster-2 (Salsa) spacecraft as a function of time and frequency. The colour scaling shown on the right-hand side gives the strength of the electric signals, which is expressed in decibels above $10^{-8} \mathrm{~V}_{\mathrm{rms}} \mathrm{Hz}^{-1 / 2}$. This means that $100 \mathrm{~dB}$ corresponds to an electric field of $1 \mathrm{~m}$ $\mathrm{V}_{\mathrm{rms}} \mathrm{Hz}^{-1 / 2}$. Cluster-2 was in the Earth's magnetosphere not far from the plasmapause, actually on the dayside at about 3 Earth radii, almost along the Earth-Sun line. As expected in this region, $n F_{c e}$ and $F_{q n}$ are actually observed, as we will see in the next section.

In the solar wind and magnetosheath, where the electron temperature and the magnetic field are low, only one strong resonance is usually observed close to the plasma frequency, from which the total plasma density is deduced. A bow shock crossing detected on board Cluster-1 (Rumba) is shown in the bottom two panels of Fig. 2. Cluster-1 was moving from the magnetosheath to the solar wind and the bow shock transition region is seen between approximately 11:10 and 12:10 UT. 

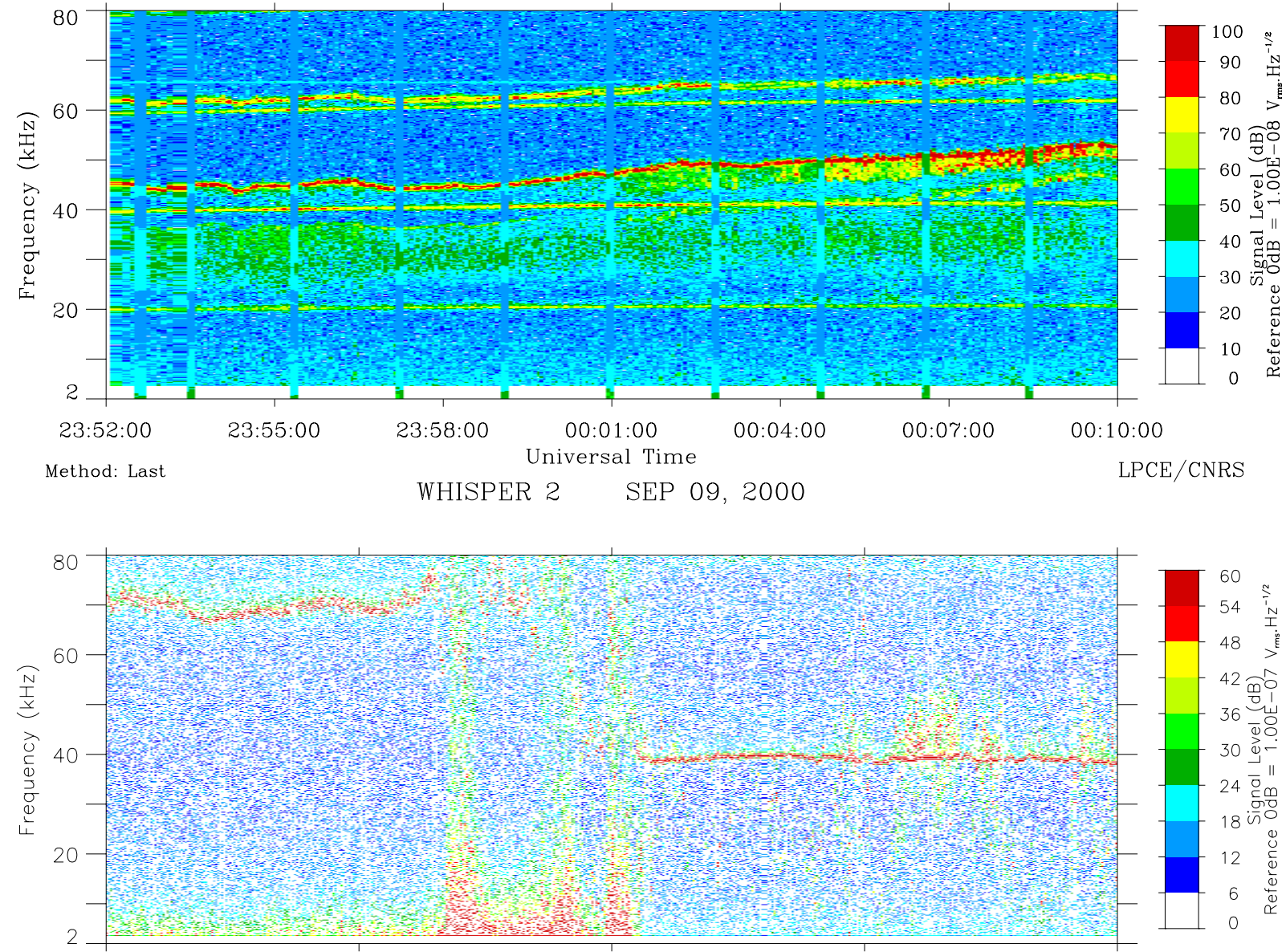
$10: 00: 00$
$11: 00: 00$
$12: 00: 00$
$13: 00: 00$
$14: 00: 00$
Method: AvgAct
Universal Time
LPCE/CNRS

\section{WHISPER 1 DEC 22, 2000}

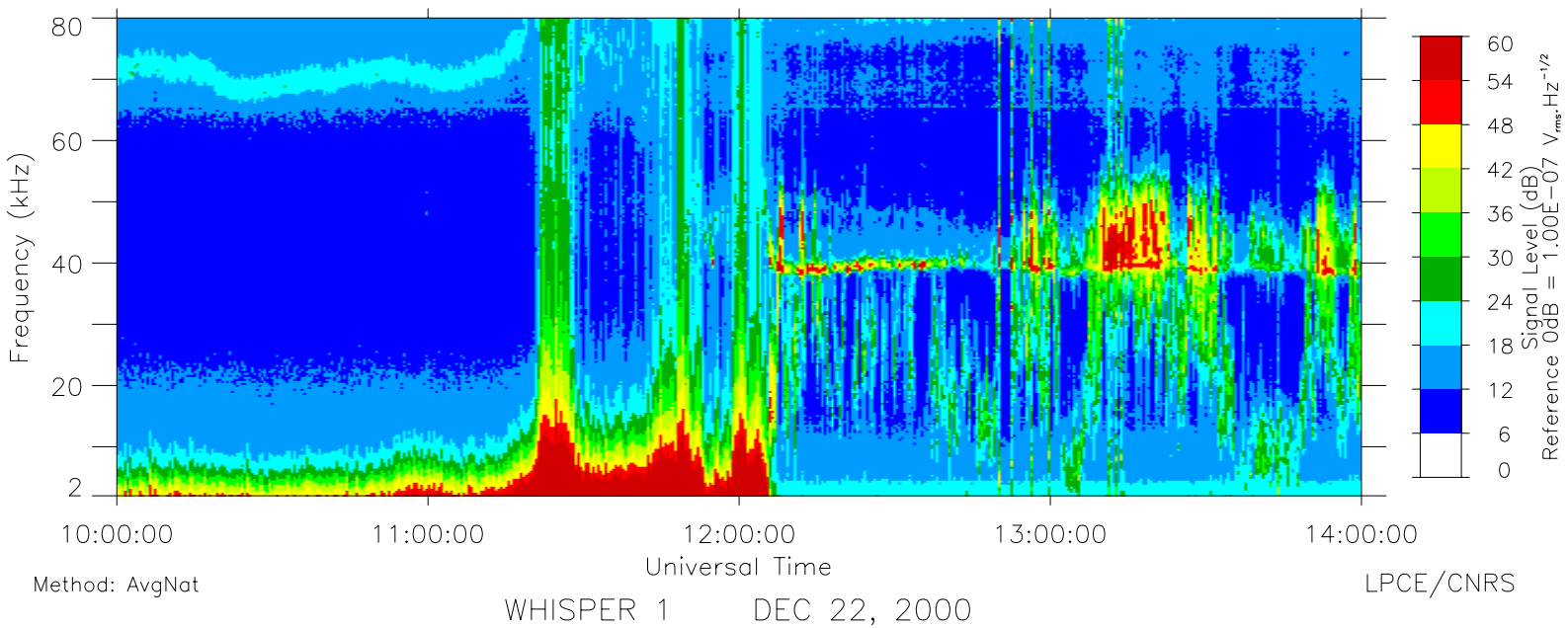

Fig. 2. Dynamic spectrograms of the electric field measured by the Whisper relaxation sounder on board Cluster-2 in the Earth's magnetosphere (top panel) and on board Cluster-1 during a magnetosheath-solar wind pass (middle and bottom panels). Active spectra are displayed in the top two panels, while passive spectra are shown in the bottom panel.

The shock was crossed in the dusk sector at $\sim 16: 50$ LT. The middle panel in Fig. 2 represents the electric field spectra triggered by Whisper when it was in its active mode, while the bottom panel exhibits the passive spectra acquired at the 


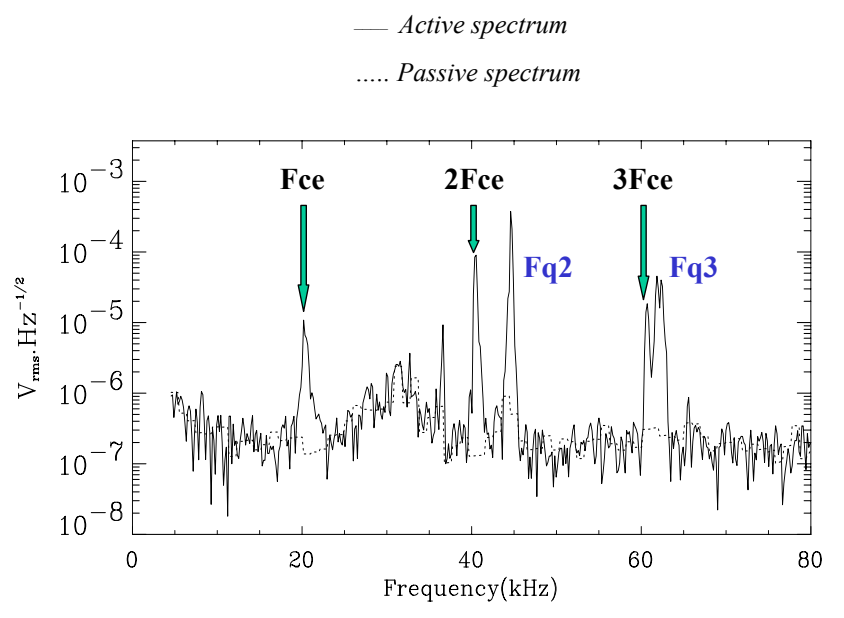

WHISPER 2 S Sept 2000, 23:58:24 UT

Fig. 3. Individual active spectrum measured in the Earth's magnetosphere by Whisper-2. Two monochromatic resonance families are clearly seen: the electron cyclotron frequency, $F_{c e}$, and two of its harmonics, $2 F_{c e}$ and $3 F_{c e}$, and two double Bernstein's mode resonances, $F_{q 2}$ and $F_{q 3}$.

same times. It becomes clear from the bottom two spectrograms in Fig. 2 that only one strong resonance is actually excited in the solar wind and in the magnetosheath, as expected.

Whenever resonances are observed and properly identified, the total plasma density, $N_{e}$, and sometimes the magnetic field strength, $B$, should then be reliably and accurately determined. $N_{e}$ and $B$ are indeed given by the well-known formulae: $N_{e}=F_{p e}^{2} / 81$ and $B=F_{c e} / 28$, where $N_{e}$ is expressed in $\mathrm{cm}^{-3}, F_{p e}$ in $\mathrm{kHz}, B$ in $\mathrm{nT}$, and $F_{c e}$ in $\mathrm{Hz}$. For example, let us come back to the magnetospheric pass shown in the middle panel of Fig. 2. At the beginning of the period, when the spacecraft is in the magnetosheath, the plasma frequency resonances are observed between $70 \mathrm{kHz}$ and $80 \mathrm{kHz}$. Consequently, the total plasma density appears to vary from $\sim 60 \mathrm{~cm}^{-3}$ to $80 \mathrm{~cm}^{-3}$. Then, from $12: 10$ UT to the end of the period, Cluster- 1 stays in the solar wind and the plasma frequency remains almost constant at a little bit less than $40 \mathrm{kHz}$, which corresponds to $\sim 19 \mathrm{~cm}^{-3}$. Let us remark that in these regions, the magnetic field is too low for the $n F_{c e}$ gyroharmonics to be observed. $F_{c e}$ is indeed of the order of the Whisper frequency resolution $(162 \mathrm{~Hz}$ in width frequency bins).

The choice of the Whisper frequency range, from 4 to $83 \mathrm{kHz}$, is based on the observations made by the relaxation sounder on board ISEE-1 that encountered similar regions. Densities within the $0.2-85 \mathrm{~cm}^{-3}$ range can thus be measured.

Before going on with resonance recognition processes, let us recall some basic knowledge of the comparative lengths of the waves excited by sounders, $\lambda$, and spherical double probes, $l$. The waves excited by resonance sounders are

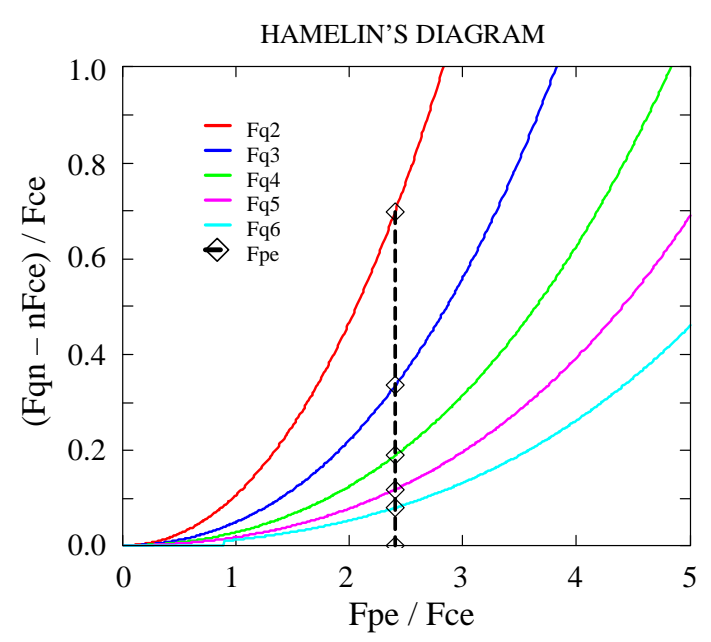

Fig. 4. Frequency positions of five of the Bernstein's mode resonances, from $F_{q} 2$ to $F_{q} 6$. The frequency separation between a $F_{q n}$ and the harmonic of the electron cyclotron frequency, $n F_{c e}$, that is just below $F_{q n}$, is plotted as a function of the plasma frequency, $F_{p e}$. For convenience, this separation and $F_{p e}$ are normalized to $F_{c e}$. The diamonds aligned on a vertical line give the $F_{q n}$ frequencies expected for a Maxwellian plasma with a given $F_{p e}$.

predominantly electrostatic in nature; their wavelengths are, therefore, most of the time, very large compared to the antenna lengths. Nevertheless, such quasi-electrostatic waves can sometimes have rather short wavelengths. Whenever $\lambda$ is close to $l$ or shorter, the antenna response must differ from the usually considered long wavelength limit. The amplitude of the received signal should vary as $\lambda$ for spherical double probes and the signal fades whenever $l$ is a multiple of $\lambda$ (Gurnett, 1998). It is worth noting that the minimum wavelength in a collective plasma is the Debye length $\lambda_{D}\left(\lambda_{D}=6.9 \sqrt{ }\left(T_{e} / N_{e}\right)\right.$, where $\lambda_{D}$ is expressed in $\mathrm{cm}$, the electron temperature $T_{e}$ in $K$, and the electron density $N_{e}$ in $\mathrm{cm}^{-3}$ ). Let us also note that in any case, the antenna must be longer than $\lambda_{D}$ in order to sense the plasma presence instead of responding as if it were in free space.

As an example, let us consider the cusp event reported by Perraut et al. (1990). Measurements performed by the relaxation sounder and the mutual impedance probe on board the Viking spacecraft have led to the following plasma parameters: $N_{e}=58 \mathrm{~cm}^{-3}$, the electron plasma frequency to gyrofrequency ratio $F_{p e} / F_{c e}=1.25$, the electron Larmor radius $r_{L}=4.8 \mathrm{~m}\left(r_{L}=V_{t h} / 2 \pi F_{c e}\right.$, where the electron thermal velocity $V_{t h}=\sqrt{ }\left(K T_{e} / m\right), K$ is the Boltzmann constant, and $m$ is the electron mass; note that $K T_{e}=15 \mathrm{eV}$ ). The Debye length can be deduced from $F_{p e} / F_{c e}$ and $r_{L}$, $\lambda_{D}=r_{L} F_{c e} / F_{p e}=3.8 \mathrm{~m}$, which has to be compared to the $80 \mathrm{~m}$ of the Viking spherical double probe length. The wavelengths of the Bernstein's resonances $F_{q 2}, F_{q 3}$, and $F_{q 4}$ can also be calculated; they are equal to $32 \mathrm{~m}, 17 \mathrm{~m}$, and > $9 \mathrm{~m}$ (see $F_{q n}$ dispersion diagram in Tataronis and Crawford, 1970), respectively. Although these wavelengths are shorter 


\section{RECOGNIZED RESONANCES}
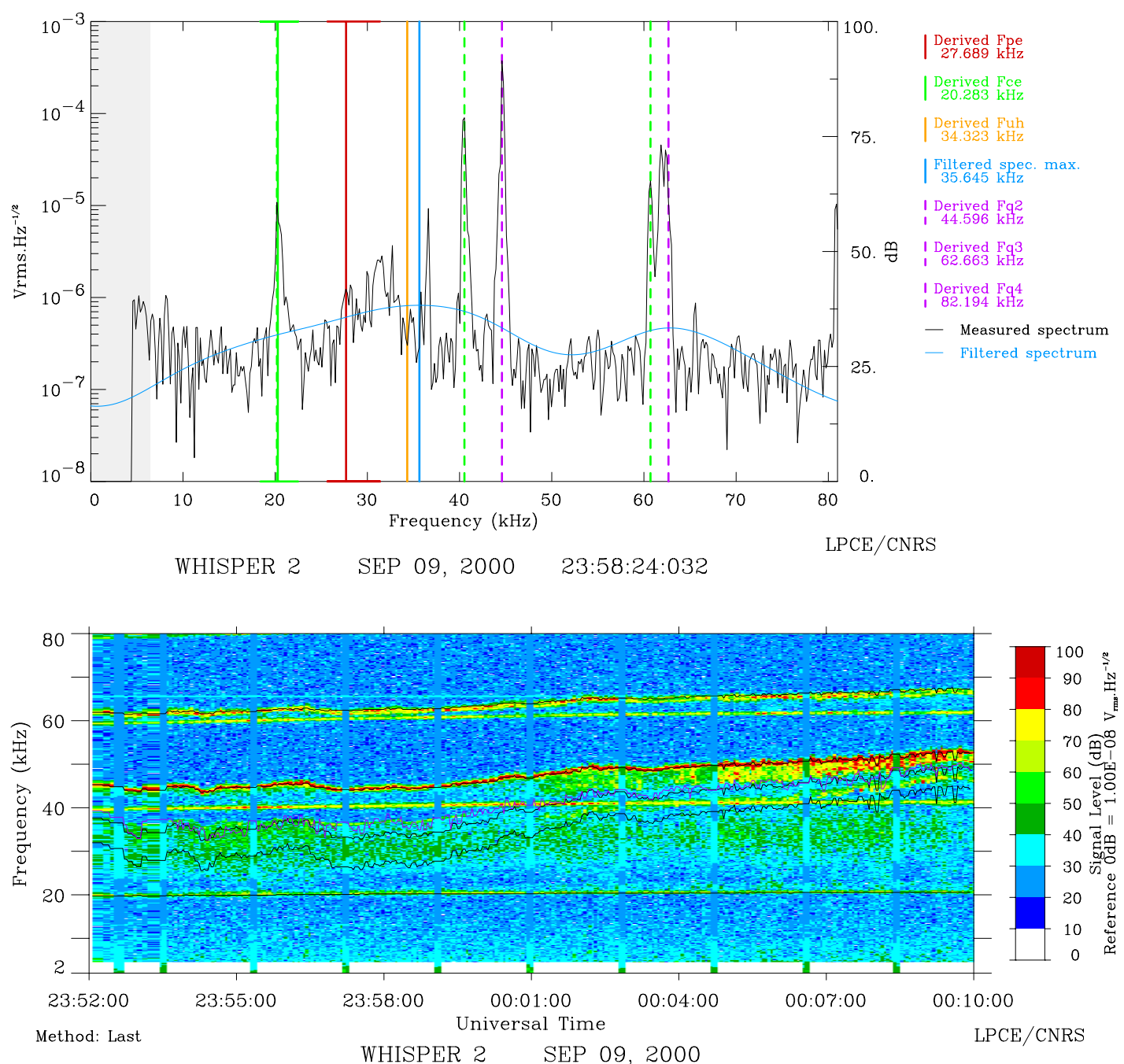

Fig. 5. Results of the resonance recognition process for a magnetospheric pass (bottom) and one of the individual spectra (top). See explanations in the text. Cluster- 2 was on the dayside, at about 3 Earth radii close to the noon direction.

than the antenna length, they were clearly seen by the Viking sounder (Perraut et al., 1990).

\section{Resonance recognition process}

The method used in the solar wind and magnetosheath, where only one strong resonance is usually excited, is rather simple whenever the resonance intensity exceeds the ambient noise level. It consists of removing the natural noise (passive spectrum) and possible interferences from the excited signal (active spectrum) and then retaining the most intense frequency bin in each resulting spectrum. It is worth noting that this method strongly differs from the pattern recognition method applied to the solar wind data recorded by the ISEE-1 relaxation sounder (Thouvenin and Trotignon, 1980), since pattern recognition methods require more information on the resonance signal than the intensity delivered by Whisper. Three parameters, the power, the time decay, and the deviation from the average resonance, were indeed necessary to best describe the ISEE-1 resonance signals.

The magnetospheric case is more intricate. Here, the problem is no longer the extraction of resonances, but the naming of them (Trotignon et al., 1986). The most easily identified resonances are the $n F_{c e}$, due to their harmonicity. The magnetometer (Balogh et al., 1997) provides $B$ and hence, the initial $F_{c e}$ value of an iterative process, which consists in correlating a computed series of gyroharmonics with the Whisper observations ( $F_{c e}=e B /(2 \pi m)$, where $e$ and $m$ are, respectively, the electron charge and mass). The returned $F_{c e}$ value of the gyroharmonic series is the one that gives the best correlation coefficient. The $F_{c e}$ and thus $B$ are usually determined with an accuracy better than $1 \%$. Figure 3 displays an individual active spectrum recorded at 23:58:24 UT on 
RECOGNIZED RESONANCES
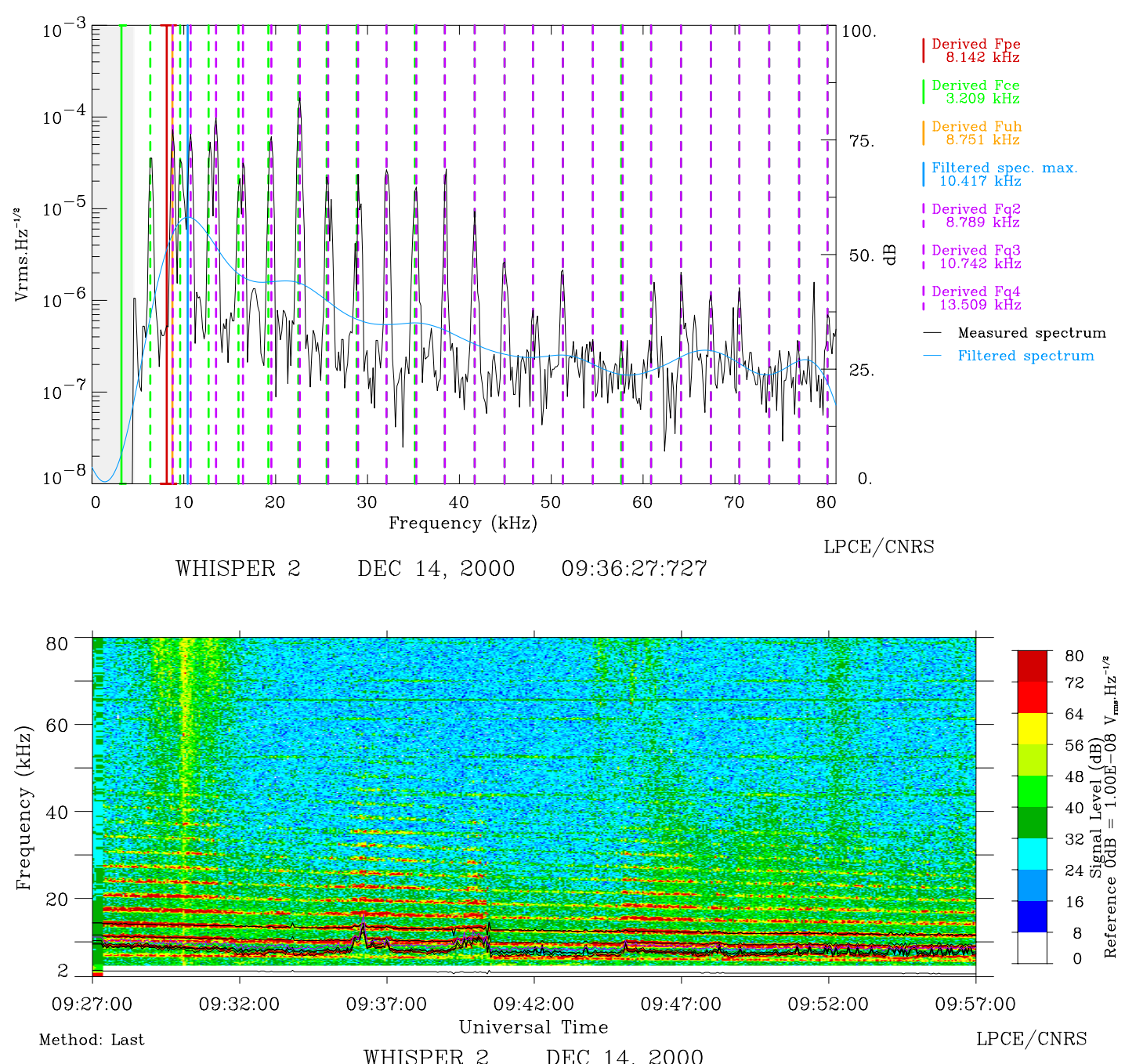

Fig. 6. Results of the resonance recognition process for a magnetospheric pass (bottom) and one of the individual spectra (top). See explanations in the text. Cluster-2 was located at $X_{\mathrm{GSE}}=-2.7 R_{E}, Y_{\mathrm{GSE}}=2 R_{E}, Z_{\mathrm{GSE}}=7.3 R_{E}$.

9 September 2000 by Whisper- 2 on board Cluster-2. It is one of the spectra that makes up the spectrogram shown in the upper panel of Fig. 2. The electron cyclotron frequency is close to $20 \mathrm{kHz}$ and the two harmonics are also seen at $\sim 40 \mathrm{kHz}$ and $60 \mathrm{kHz}$. It allows for the magnetic field strength to be estimated at a little bit more than $700 \mathrm{nT}$.

A second resonance family, the $F_{q n}$, is usually provided by active sounding. For example, two spikes (resonances) or more precisely, double spikes are actually triggered round $45 \mathrm{kHz}$ and $63 \mathrm{kHz}$ in Fig. 3. This second resonance family may be identified by comparing the dispersion relation of the Bernstein's electrostatic waves to the measured spectra. Again, the $F_{q n}$ series that gives the best correlation coefficient allows for an accurate plasma frequency and thus, an absolute plasma density to be delivered $\left(2 \pi F_{p e}=\right.$ $\sqrt{ }\left(N_{e} e^{2} / m \varepsilon_{o}\right)$, where $\varepsilon_{o}$ is the free space dielectric constant). The $F_{q n}$ series indeed only depends on the $F_{p e} / F_{c e}$ ratio for a Maxwellian plasma. Their frequency locations are conveniently given by the so-called Hamelin's diagram that is discussed in Sect. 4.

Closely linked to this process is the way one determines the $F_{p e}$ initial value, when $F_{c e}$ is known. We have established from previous observations that the resonances are more intense both close to and above the plasma frequency (Trotignon et al., 1986). This means that an upper estimate of $F_{p e}$ may be derived from the maximum intensity of the smoothed spectrum simply obtained by applying a low-pass filter to the measured spectrum. This point is illustrated in Sect. 5.

\section{Hamelin's diagram}

In the Hamelin's diagram exhibited in Fig. 4, the frequencies are normalized to $F_{c e}$. Each curve labelled $F_{q n}$, where $n$ is 

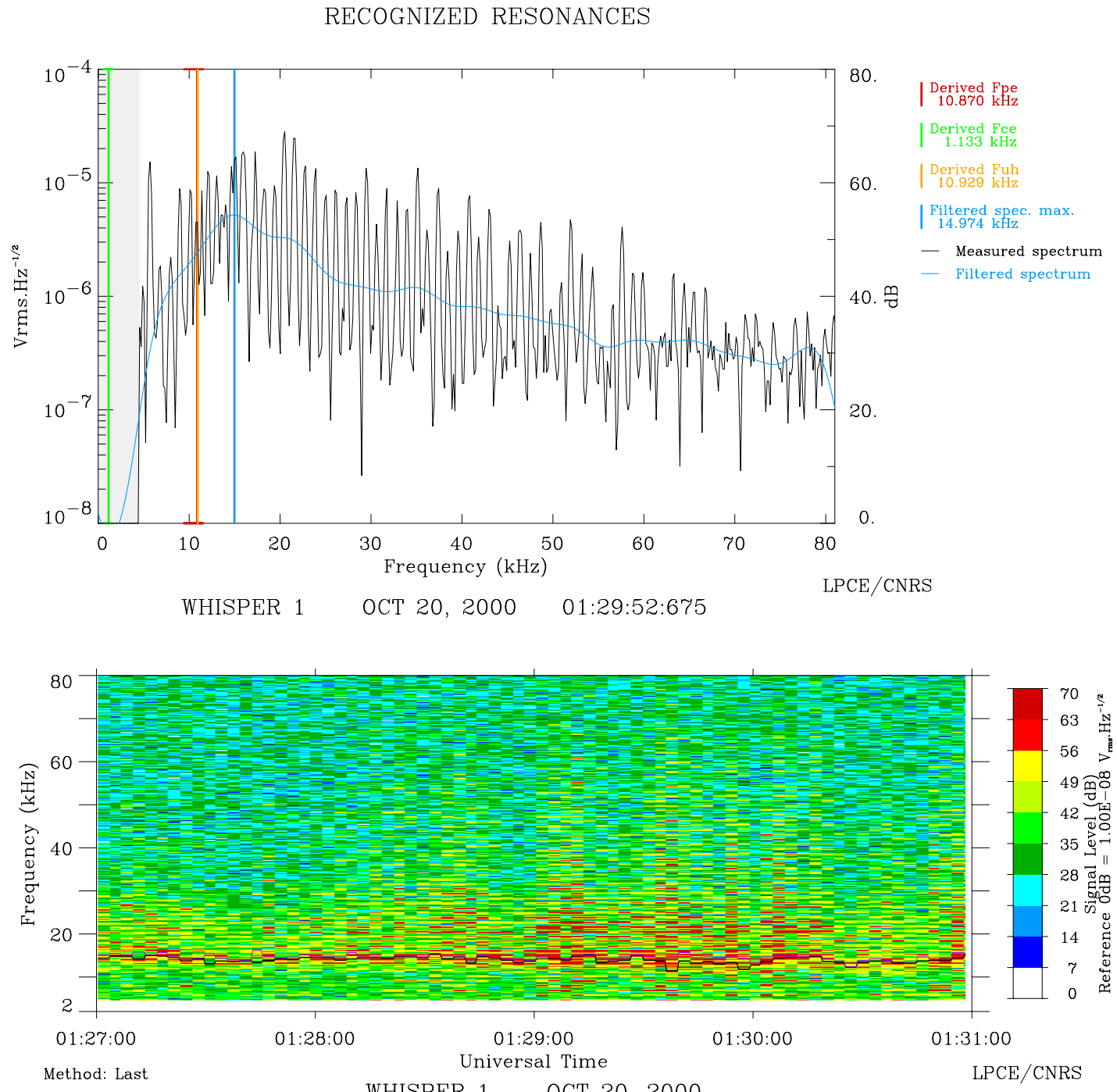

Fig. 7. Results of the resonance recognition process for a magnetospheric pass (bottom) and one of the individual spectra (top). See explanations in the text. Cluster-1 was located at $X_{\mathrm{GSE}}=-6 R_{E}, Y_{\mathrm{GSE}}=10.5 R_{E}, Z_{\mathrm{GSE}}=-6.5 R_{E}$.

of the order of the $n F_{c e}$ gyroharmonic that is just below $F_{q n}$, represents the frequency interval between $F_{q n}$ and $n F_{c e}$ normalized to $F_{c e}$, when $F_{p e} / F_{c e}$ varies (Hamelin, 1978; Pottelette et al., 1981).

For a given plasma frequency, $F_{p e}$, all the $F_{q n}$ appear aligned on a vertical line in this diagram. Let us point out that this is true only for a Maxwellian plasma. In non-Maxwellian plasmas, the $F_{q n}$ could be split and non-aligned, corresponding to plasma frequencies ranging from the plasma frequency of the coldest component to the total plasma frequency (Belmont, 1981). This means that a careful analysis of the spectra provided by the Whisper active sounding may provide a fine diagnosis of non-Maxwellian plasmas. Belmont (1981) has indeed shown that the shape of the electron distribution function modifies the Bernstein's electrostatic wave dispersion curves of the Bernstein's electrostatic waves. In other words, when the $F_{q n}$ resonances appear aligned in the Hamelin's diagram, the plasma distribution function may be considered as
Maxwellian, while non-aligned $F_{q n}$ resonance series should be the signature of several plasma components. It is then possible to assume that the distribution function is composed of two Maxwellian components and the expected $F_{q n}$ frequency loci fit the observed ones. Such sophisticated analysis is out of scope for this paper, but will be investigated in further studies.

\section{Resonance recognition results}

A program to automatically determine the plasma density from the observed plasma resonances is under development. The technique used is described above. It implies that one automatically determine the encountered region, or at least one reasonably anticipates the number of plasma characteristic frequencies and which categories they belong to. This can be done thanks to the spacecraft location data, the measured 
magnetic fields (Balogh et al., 1997), and a magnetosphere model (Kosik, 1998). We have to know if the spacecraft is either in the solar wind, the magnetosheath, the outer magnetosphere, the plasmasphere, the cusp regions, or in the far tail. Although it is not positively required, the diagnosis is more precise and reliable whenever the encountered region is known and the appropriate resonance recognition process is applied. Here are some results for the different $F_{p e} / F_{c e}$ ratios.

The bottom panel in Fig. 5 shows the recognized resonances superimposed on the electric field spectra measured by Whisper on 9 September 2000. This measured spectrogram is the same as the one that is shown at the top of Fig. 2. The lowest black line, close to $20 \mathrm{kHz}$, is the recognized electron gyrofrequency, $F_{c e}$. The second black line, just above $F_{c e}$, stands for the computed plasma frequency, $F_{p e}$. It is obtained from the best fit of the theoretical Berntein's mode frequencies, $F_{q n}$, which are aligned in the Hamelin's diagram, to the observed $F_{q n}$ resonances. The upper hybrid frequency, $F_{u h}$, derived from the computed $F_{c e}$ and $F_{p e}$ values, is shown by the third black line, while the upper two black lines are the $F_{q 2}$ and $F_{q 3}$ given by the program. Finally, the maximum intensities of the smoothed spectra are plotted in purple. Except for some erroneous identifications at the end of the period, the program turns out to work satisfactorily. As $F_{p e}$ and $F_{c e}$ are found to be $25-45 \mathrm{kHz}$ and $20 \mathrm{kHz}$, respectively, we can conclude that in this region, the plasma density and magnetic field modulus are of the order of $8-25 \mathrm{~cm}^{-3}$ and $700 \mathrm{nT}$, respectively.

An individual spectrum acquired at 23:58:24 UT on the same day (this spectrum is also shown in Fig. 3) is displayed at the top of Fig. 5 with the computed resonances. $F_{p e}$ and $F_{c e}$ are found to be $27.7 \mathrm{kHz}$ (red vertical line) and $20.3 \mathrm{kHz}$ (green vertical solid line), respectively; these values correspond to a density of $9.5 \mathrm{~cm}^{-3}$ and a magnetic field intensity of $725 \mathrm{nT}$. As can be seen, the orange vertical line that gives the expected $F_{u h}$ does not coincide with the peak that is observed $2 \mathrm{kHz}$ above. Let us also remark that no strong resonance seems to be associated with the derived $F_{p e}$; this is often the case. The $F_{u h}$ and $F_{p e}$ resonances are sometimes observed, either both or separately, and sometimes they are not seen at all. Further investigations will be necessary in order to comment further about these behaviours. Finally, the blue vertical line is the maximum of the smoothed spectrum that is represented as a blue wavy line, and the two violet vertical dashed lines are the obtained $F_{q 2}$ and $F_{q 3}$.

Figures 6 and 7 confirm that the resonance recognition process works efficiently in the magnetosphere when $F_{c e}$ is small $\left(3.2 \mathrm{kHz}\right.$ and $1.1 \mathrm{kHz}$, respectively,) and $F_{p e} / F_{c e}$ is either low or high ( 2.5 and 9.6, respectively). Incidentally, it is important to note here that the smoothed spectrum maximum (blue vertical line in the top panels of Figs. 6 and 7) is always above the plasma frequency (red vertical line in Fig. 6 and orange line in Fig. 7, the latter actually covers the red one). Let us recall that the frequency location of this maximum is used as an initial value (upper value) for the plasma frequency determination process (see Sect. 3).
On 24 September 2000, during the spacecraft commissioning phase, Cluster- 2 crossed the plasmapause twice, round 07:05 UT and 08:19UT. The resonances triggered by Whisper-2 around 08:19 UT are shown in the top panel of Fig. 8, when Cluster-2 was leaving the Earth's plasmasphere and it entered the magnetosphere at 08:22 UT. At 08:19UT, salsa was located at $X_{\mathrm{GSE}}=3.16 R_{E}, Y_{\mathrm{GSE}}=-2.0 R_{E}$, $Z_{\mathrm{GSE}}=1.6 R_{E}$, where $R_{E}$ is the Earth's radius and GSE denotes the geocentric solar ecliptic coordinate system. The $L$ and invariant latitude were 5.3 and $64.4^{\circ}$, respectively, which is common for the dayside plasmapause (Chappell et al., 1971). The purple lines connect the observed $2 F_{c e}, 3 F_{c e}$, and $4 F_{c e}$ resonances; the green diamonds stand for the $F_{q 2}$, $F_{q 3}$, and $F_{q 4}$; the red triangles represent $F_{u h}$, while the black stars denote the frequency location of the resonance called $F_{x}$ by Belmont (1981). Finally, the black crosses are the $F_{u h}$ frequency calculated from the $F_{c e}$ and $F_{x}$ measured values. As can be seen, black crosses and red triangles almost merge, as expected, if $F_{x}$ is the plasma frequency. The electron density derived from the $F_{x}$ and $F_{u h}$ observations is also displayed as a function of time in the bottom panel of Fig. 8.

The resonance recognition technique described above has been applied to the 24 September 2000 plasmapause crossing event shown in Fig. 8. The recognized resonances are displayed at the top of Fig. 9: the quasi-horizontal red lines connect the calculated gyroharmonics $n F_{c e}$ and the green diamonds denote the calculated Bernstein's resonances $F_{q n}$. Although several Bernstein's resonances are actually observed between two successive harmonics of $F_{c e}$, as shown in the top panel of Fig. 8, only one is given by the resonance recognition programme, since we are looking for the series of Bernstein's resonances that are aligned in the Hamelin's diagram. The relative plasma frequency, here called $F_{a l}$ for convenience, and the associated upper-hybrid frequency $F_{u h}$ are plotted as black stars and purple triangles, respectively, in the top panel of Fig. 9. The blue crosses stand for the frequency locations of the smoothed spectrum maxima, which are used as upper estimates of the plasma frequency in the automatic recognition process. Three estimates of the electron plasma density can thus be provided; they are plotted in the bottom panel of Fig. 9. The black line is the density calculated from the alignment of the Bernstein's resonances in the Hamelin's diagram, the blue line is the density given by the smoothed spectrum maxima, and the red stars are the densities deduced from the $F_{x}$ observations. The latter curve is similar to the one that is plotted as a black line at the bottom of Fig. 8. As can be seen, the assumption that the smoothed spectrum maxima occur most of the time above the plasma frequency is valid. Another significant result is that the $F_{a l}$ value derived from the Bernstein's resonances alignment in the Hamelin's diagram is always below the observed $F_{x}$. It is worth recalling that Belmont (1981) and Etcheto et al. (1983) have demonstrated that $F_{a l}$ corresponds to the cold electron population, while $F_{x}$ relates to the total electron population, the latter resonance being the only one actually observed. This is corroborated here by the existence of a non-aligned Bernstein's resonance series (not shown in the paper). Al- 

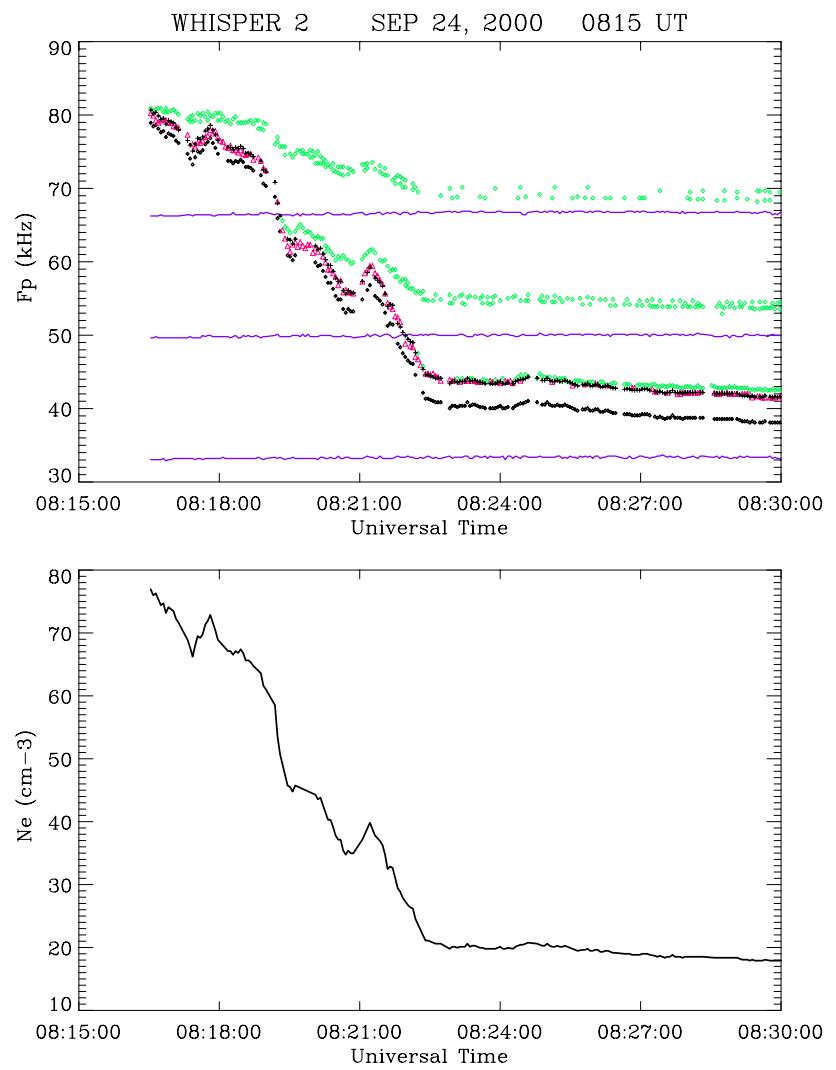

Fig. 8. Frequency of the resonances observed by Whisper-2 during an outbound crossing of the Earth's plasmapause as a function of time (top). Electron plasma density is deduced from the observed plasma frequency and upper-hybrid frequency resonances (bottom). See details in the text.

though this point is beyond the scope of this presentation, we may say that the apparent percentage of the cold electrons is found to be $95 \%$ in the plasmasphere and $60 \%$ in the magnetosphere. These results have to be confirmed by particle experiment measurements; unfortunately, these instruments were not switched on during this phase of the Cluster commissioning. Such comparison will be performed when the four Cluster spacecraft encounter again the same region. Finally, let us note that the frequency at which the smoothed spectrum amplitude is maximum appears to be very close to the total upper-hybrid frequency that is actually observed.

\section{Summary and conclusions}

The Whisper relaxation sounder is primarily an active experiment, aimed at exciting radio waves at the plasma characteristic frequencies, thus providing information on the local plasma. As a resonance sounder, Whisper indeed reliably determined the total electron density and when the electron cyclotron frequency is of the order of $1 \mathrm{kHz}$ or more, the magnetic field modulates.
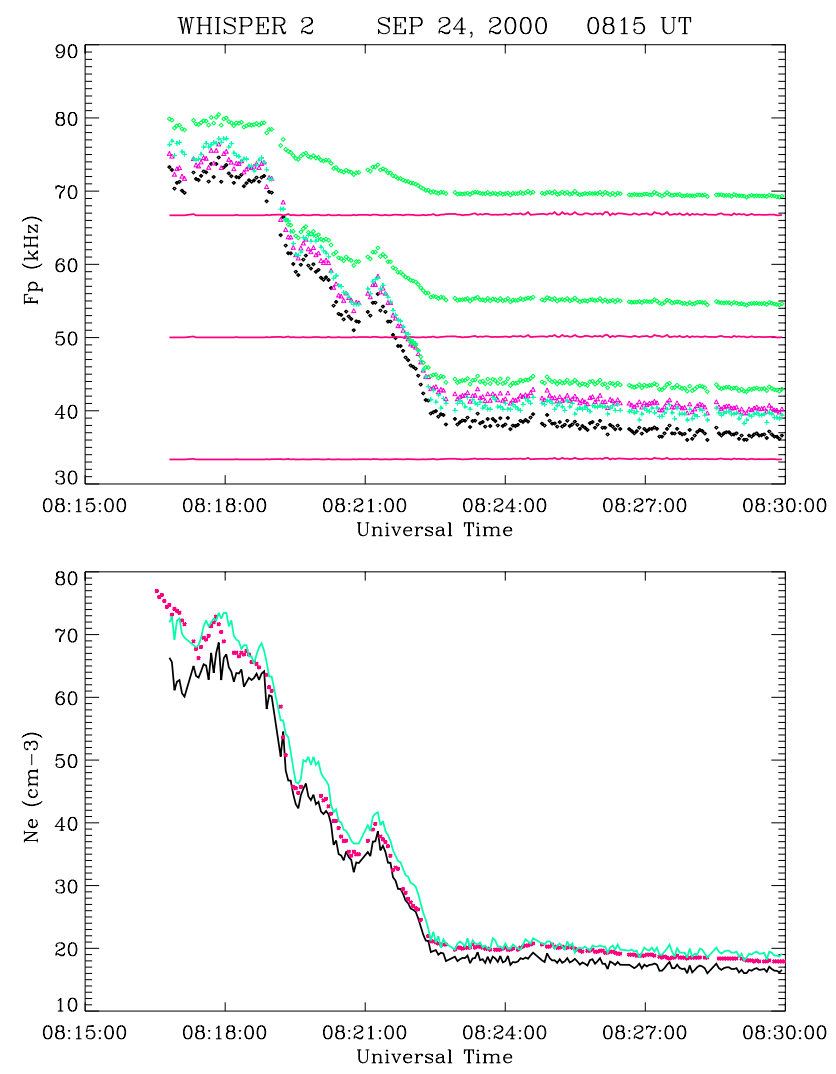

Fig. 9. Frequency of the recognized/calculated resonances (top) and inferred electron plasma densities (bottom) versus time. For comparison, the observed resonances are shown in Fig. 8. The red stars in the bottom panel represent the observed total plasma density. For further details, see the text.

An automatic method for deriving these fundamental plasma parameters is under development. In the solar wind and magnetosheath, only one strong resonance is usually observed at the plasma frequency. A simple algorithm looks for the most intense frequency bin inside each active spectrum. In the magnetosphere, where different types of resonances occur, the harmonics of the electron cycloton frequency, $n F_{c e}$, are first identified by taking into account their harmonicity. The Bernstein's mode frequencies, $F_{u h}$ and $F_{q n}$, are, in turn, determined from the theoretical dispersion relation of the Bernstein's modes. Then, the plasma frequency and hence, the plasma density, are deduced from the alignment of the $F_{q n}$ in the Hamelin's diagram, at least when the plasma distribution function is more or less Maxwellian and the plasma is almost stationary over the time required to obtain a full spectrum (less than $2 \mathrm{~s}$ ). If this is not the case, then the $F_{q n}$ resonances may be divided into two non-aligned series: a more sophisticated analysis is then possible, which should give valuable information about the plasma components that are actually present. A hot electron component may be indeed detected and its density, and possibly temperature, estimated. Further works will be done in this direction in the immediate future. 
Finally, it is worth pointing out that a careful and accurate determination of the characteristic frequencies of the encountered plasmas is fundamental to interpreting observed natural waves. This is, for example, the only way to reliably identify cutoff frequencies or wave excitation maxima and hence, to determine the modes in which the natural waves are actually propagating (see, for example, Décréau et al., 2001, this issue and Canu et al., 2001, this issue). We must also keep in mind that the density determination that relies on natural waves or spacecraft potential measurements cannot be considered as completely reliable. As said above, a misinterpretation is often possible when no cross-check with an active experiment is done.

Acknowledgement. We are very grateful to E. Guyot, L. Launay, and J.-L. Fousset who have developed most of the Whisper data processing tools. We would like also to thank H. Poussin, M. NononLatapie, D. Delmas, H. Marquier, K. Amsif, D. Gras, J.-P. Thouvenin, and J.-Y. Prado from CNES for their effective support.

The Editor in Chief thanks two referees for their help in evaluating this paper.

\section{References}

Balogh, A., Dunlop, M. W., Cowley, S. W. H., Southwood, D. J., Thomlinson, J. G., Glassmeier, K. H., Musmann, G., Lühr, H., Buchert, S., Acunã, M. H., Fairfield, D. H., Slavin, J. A., Riedler, W., Schwingenschuh, K., Kivelson, M. G., and the Cluster magnetometer team: The Cluster magnetic field investgation, Space Sci. Rev., 79, 65-91, 1997.

Belmont, G.: Characteristic frequencies of a non-Maxwellian plasma: a method for localizing the exact frequencies of magnetospheric intense natural waves near $F_{p e}$, Planet. Space Sci., 29, 1251-1266, 1981.

Belmont, G., Canu, P., Etcheto, J., de Feraudy, H., Higel, B., Pottelette, R., Béghin, C., Debrie, R., Décréau, P. M. E., Hamelin, M., and Trotignon, J. G.: Advances in magnetospheric plasma diagnosis by active experiments, in Proc. Conf. Achievements of the IMS, 26-28 June 1984, Graz, Austria, ESA SP-217, 695699, 1984.

Bernstein, I. B.: Waves in a plasma in a magnetic field, Phys. Rev., 109, 10-21, 1958.

Canu, P., Décréau, P., Trotignon, J. G., Rauch, J. L., Séran, H. C., Fergeau, P., Lévêque, M., Martin, Ph., Sené, F. X., Le Guirriec, E., Alleyne, H., and Yearby, K.: Identification of natural plasma emissions with the Whisper relaxation sounder, Ann. Geophysicae, 2001, this issue.

Chappell, C. R., Harris, K. K., and Sharp, G. W.: The dayside of the plasmasphere, J. Geophys. Res., 76, 7632-7647, 1971.

Décréau, P. M. E., Fergeau, P., Krasnoselskikh, V., Lévêque, M., Martin, Ph., Randriamboarison, O., Sené, F. X., Trotignon, J. G., Canu, P., Mögensen, P. B., and Whisper Investigators: Whisper, a resonance sounder and wave analyser: performances and perspectives for the Cluster mission, Space Sci. Rev., 79, 157-193, 1997.

Décréau, P. M. E., Fergeau, P., Krasnoselskikh, V., Le Guirriec,
E., Lévêque, M., Martin, Ph., Randriamboarison, O., Rauch, J. L., Sené, F. X., Séran, H. C., Trotignon, J. G., Canu, P., Cornilleau, N., De Féraudy, H., Alleyne, H., Yearby, K., Woolliscroft, L., Mögensen, P. B., Gustafsson, G., André, M., Gurnett, D. C., Darrouzet, F., and Whisper experimenters: Early results from the Whisper instrument on Cluster: an overview, Ann. Geophysicae, 2001, this issue.

Etcheto, J., de Feraudy, H., and Trotignon, J. G.: Plasma resonance stimulation in space plasmas, Adv. Space Res., 1, 183196, 1981.

Etcheto, J., Belmont, G., Canu, P., and Trotignon, J. G.: Active sounder experiments on GEOS and ISEE, in Active experiments in space symposium, 24-29 May 1983, Alpbach, ESA SP-195, 39-46, 1983.

Gurnett, D. A.: Principles of space plasma wave instrument design, in: Measurement techniques in space plasmas: fields, (Eds) Pfaff, R. F., Borovsky, J. E., and Young, D. T., American Geophysical Union, USA, pp. 121-136, 1998.

Gustafsson, G., Boström, R., Holback, B., Holmgren, G., Lundgren, A., Stasiewicz, K., Åhlén, L., Mozer, F. S., Pankow, D., Harvey, P., Berg, P., Ulrich, R., Pedersen, A., Schmidt, R., Butler, A., Fransen, A. W. C., Klinge, D., Thomsen, M., Fälthammar, C.-G., Lindqvist, P.-A., Christenson, S., Holtet, J., Lybekk, B., Sten, T. A., Tanskanen, P., Lappalainen, K., and Wygant, J.: The electric field and wave experiment for the Cluster mission, Space Sci. Rev., 79, 137-156, 1997.

Hamelin, M.: Contribution à l'étude des ondes électrostatiques et électromagnétiques au voisinage de la fréquence hybride basse dans le plasma ionosphérique, Thèse de Doctorat d'Etat, Université d'Orléans, Orléans, France, 1978.

Kosik, J. C.: A quantitative model of the magnetosphere with poloidal vector fields, Ann. Geophysicae, 16, 1557-1566, 1998.

Lefeuvre, F., Roux, A., de la Porte, B., Dunford, C., Woolliscroft, L. C. C., Davies, P. N. H., Davis, S. J., and Gough, M. P.: The Wave Experiment Consortium, in Cluster: mission, payload and supporting activities, ESA SP-1159, 5-15, 1993.

Perraut, S., de Feraudy, H., Roux, A., Décréau, P. M. E., Paris, J., and Matson, L.: Density measurements in key regions of the Earth's magnetosphere: cusp and auroral region, J. Geophys. Res., 95, 5997-6014, 1990.

Pottelette, R., Hamelin, M., Illiano, J. M., and Lembège, B. L.: Interpretation of the fine structure of electrostatic waves excited in space, Phys. Fluids, 24, 1517-1526, 1981.

Tataronis, J. A. and Crawford, F. W.: Cyclotron harmonic wave propagation and instabilities: I. perpendicular propagation, J. Plasma Phys., 4, 231-248, 1970.

Thouvenin, J. P. and Trotignon, J. G.: Automatic resonance recognition method in solar wind, Nuovo Ciment. Soc. Ital. Fis., C, 3, 696-710, 1980.

Trotignon, J. G., Etcheto, J., and Thouvenin, J. P.: Automatic determination of the electron density measured by the relaxation sounder on board ISEE-1, J. Geophys. Res., 91, 4302-4320, 1986.

Woolliscroft, L. J. C., Alleyne, H. ST. C., Dunford, C. M., Sumner, A., Thomson, J. A., Walker, S. N., Yearby, K. H., Buckley, A., Chapman, S., Gough, M. P., and the DWP Co-Investigators: The digital wave-processing experiment on Cluster, Space Sci. Rev., 79, 209-231, 1997. 